\title{
$\alpha$-Aminoadipic acid protects against retinal disruption through attenuating Müller cell gliosis in a rat model of acute ocular hypertension
}

This article was published in the following Dove Press journal:

Drug Design, Development and Therapy

20 October 2016

Number of times this article has been viewed

\author{
Xiaolei Wang, ${ }^{1,2, *}$ Jier Su, ${ }^{2,3, *}$ \\ Jingwen Ding, ${ }^{4}$ Song Han, ${ }^{2}$ \\ Wei Ma, ${ }^{2,5}$ Hong Luo, ${ }^{2}$ \\ Guy Hughes, ${ }^{6}$ Zhaoyang \\ Meng, 'Yi Yin,' 'Yanling \\ Wang,' Junfa $\mathrm{Li}^{2}$ \\ 'Department of Ophthalmology, \\ Beijing Friendship Hospital, \\ ${ }^{2}$ Department of Neurobiology, Beijing \\ Institute for Brain Disorders, Capital \\ Medical University, Beijing, ${ }^{3}$ Ningbo \\ College of Health Sciences, Ningbo, \\ ${ }^{4}$ Department of Ophthalmology, \\ Beijing Tongren Hospital, Capital \\ Medical University, Beijing, ${ }^{5}$ Beijing \\ Stomatological Hospital, Capital \\ Medical University, Beijing, People's \\ Republic of China; 'University of \\ California, Irvine School of Medicine, \\ Irvine, CA, USA \\ *These authors contributed equally \\ to this work
}

Objective: Ocular hypertension is an important risk factor for glaucoma. The purpose of this study was to investigate the gliotoxic effects of $\alpha$-aminoadipic acid (AAA) in a rat model of $\mathrm{AOH}$ and its underlying mechanisms.

Materials and methods: In the rat model of acute ocular hypertension (AOH), intraocular pressure was increased to $110 \mathrm{mmHg}$ for 60 minutes. Animals were divided into four groups: sham operation (Ctrl), AOH, AOH + phosphate-buffered saline (PBS), and AOH + AAA. Cell apoptosis in the ganglion cell layer was detected with the terminal deoxynucleotidyl transferasemediated uridine 5'-triphosphate-biotin nick end labeling (TUNEL) assay, and retinal ganglion cells (RGCs) immunostained with Thy-1 were counted. Müller cell activation was detected using immunostaining with glutamine synthetase and glial fibrillary acidic protein. Tumor necrosis factor- $\alpha$ (TNF- $\alpha$ ) was examined using Western blot.

Results: In the rat model of $\mathrm{AOH}$, cell apoptosis was induced in the ganglion cell layer and the number of RGCs was decreased. Müller cell gliosis in the retinas of rats was induced, and retinal protein levels of TNF- $\alpha$ were increased. Intravitreal treatment of AAA versus PBS control attenuated these retinal abnormalities to show protective effects in the rat model of $\mathrm{AOH}$.

Conclusion: In the retinas of the rat model of AOH, AAA treatment attenuated retinal apoptosis in the ganglion cell layer and preserved the number of RGCs, likely through the attenuation of Müller cell gliosis and suppression of TNF- $\alpha$ induction. Our observations suggest that AAA might be a potential therapeutic target in glaucoma.

Keywords: glaucoma, acute ocular hypertension, $\alpha$-aminoadipic acid, retina, Müller cells, retinal ganglion cells, TNF- $\alpha$

\section{Introduction}

Glaucoma, a neurodegenerative disease, is characterized by gradual loss of retinal ganglion cells (RGCs) and optic nerve atrophy. ${ }^{1}$ Multiple factors are related to glaucoma, such as high intraocular pressure (IOP) ${ }^{2}$ and low cerebral spinal fluid pressure. ${ }^{3}$ Müller cells are the major type of glial cells in the mammalian retina that can support and nourish retinal neurons, maintain extracellular ion homeostasis, glutamate recycling, and interaction in synaptic transmission. ${ }^{4,5}$ Therefore, Müller cells are involved in retinal function. Investigations indicate that Müller cells not only play an important physiological role, but they are also involved in multiple pathological retinal diseases such as glaucoma. ${ }^{6-8}$ It has been reported that reactive Müller cells can aggravate retinal damage by releasing cytokines such as tumor necrosis factor- $\alpha$ (TNF- $\alpha){ }^{9}$ Growing evidence supports that increased glial production of TNF- $\alpha$ contributes to the neurodegeneration in glaucoma. ${ }^{10} \mathrm{TNF}-\alpha$ is a secreted inflammatory cytokine that 
is responsible for apoptosis, necrosis, and inflammation. ${ }^{11,12}$ TNF- $\alpha$ is increased in the retina following ischemia or damage and other neurodegenerative disorders. ${ }^{13-17}$

$\alpha$-Aminoadipic acid (AAA), a six-carbon homolog of glutamate, is a well-known compound that induces specific glial toxicity through blocking the glutamate uptake. ${ }^{18-20}$ The use of AAA to interfere with glial influence on neuronal tissues is reported. ${ }^{21,22}$ However, the effects of AAA inhibition on Müller cell gliosis in glaucoma are unknown. The current study aimed to determine if AAA treatment inhibits Müller cell gliosis and protects against retinal abnormalities induced in a rat model of acute ocular hypertension $(\mathrm{AOH})$ mimicking glaucoma. Müller cell activation of RGCs and TNF- $\alpha$ induction were examined.

\section{Materials and methods Animals}

Adult male Sprague Dawley rats (weight range, 200-250 g, 8-10 weeks) were used in this study in accordance with the Association for Research in Vision and Ophthalmology Statement for the Use of Animals in Ophthalmic and Vision Research and followed the People's Republic of China animal care guidelines. This study was monitored and approved by the Animal Care Committee of the Capital Medical University, SCXK (Jing) 2012-0001. The animals were housed with a 12-hour light/12-hour dark cycle with standard chow and water. All surgical procedures were performed under anesthesia with intraperitoneal chloral hydrate $(450 \mathrm{mg} / \mathrm{kg})$ and topical $0.5 \%$ proparacaine hydrochloride eye drops (Alcon, Inc., Hünenberg, Switzerland).

\section{Rat model of $\mathrm{AOH}$}

The IOP was increased to $110 \mathrm{mmHg}(14.63 \mathrm{kPa})$ for 60 minutes by using an elevated $500 \mathrm{~mL}$ plastic container of sterile physiological saline connected to a 27-gauge needle placed in the anterior chamber of the eye. Sham procedure eyes were treated similarly but without the elevation of the bottle; hence, the normal IOP was maintained. Time-course examination was performed at 1 day, 3 days, and 5 days after acute IOP elevation. Animals were divided into sham operation (Ctrl) group, $\mathrm{AOH}$ group, $\mathrm{AOH}+$ phosphate-buffered saline (PBS) control group, and $\mathrm{AOH}+\mathrm{AAA}$-treated group. AAA $(250 \mu \mathrm{g})$ was injected into vitreous humor in $5 \mu \mathrm{L}$ at $50 \mathrm{~g} / \mathrm{L}$ concentration after 3 hours of acute IOP elevation according to the literature..$^{23,24}$

\section{Immunohistochemistry}

Tissues were fixed with 4\% paraformaldehyde. Eyeballs were cryoprotected in $30 \%$ sucrose overnight at $4^{\circ} \mathrm{C}$ and then embedded in optimal cutting temperature compound (Sakura Finetechinical Co. Ltd., Tokyo, Japan). Sections (14 $\mu \mathrm{m})$ were incubated in 3\% bovine serum albumin and $0.3 \%$ Triton X-100 (Sigma-Aldrich Co., St Louis, MO, USA) to block nonspecific binding and then incubated with primary mouse monoclonal antibodies against Thy-1 (1:200, RGC marker; Abcam, Cambridge, UK), primary mouse monoclonal antibodies against glutamine synthetase (GS; 1:100, Müller glial cell maker; Abcam), rabbit polyclonal antibodies against glial fibrillary acidic protein (GFAP; 1:200, gliosis maker; Cell Signaling Technology Inc., Danvers, MA, USA) overnight at $4{ }^{\circ} \mathrm{C}$, followed by incubation with secondary antibodies for 1 hour at room temperature. Nuclei were stained by Hoechst 33342. Sections were mounted with Fluoromount-G (SouthernBiotech, Birmingham, AL, USA). Negative controls were incubated without primary antibodies. Slides were visualized using a confocal microscope (Leica Microsystems, Wetzlar, Germany). The intensity of immunoreactivity from photographs was analyzed using Image-Pro ${ }^{\circledR}$ Plus 6.0 (Media Cybernetics Inc., Silver Spring, MD, USA). Optical densities obtained from immunohistochemistry images were corrected by subtracting the average value of background noise from five image inputs.

\section{Terminal deoxynucleotidyl transferase- mediated uridine $5^{\prime}$-triphosphate-biotin nick end labeling assay}

The terminal deoxynucleotidyl transferase-mediated uridine 5 '-triphosphate-biotin nick end labeling (TUNEL) assay was conducted according to the manufacturer's protocol. Briefly, tissue sections were fixed in paraformaldehyde and were subsequently incubated in a TUNEL reaction medium. The immune-labeled RGCs were costained with Hoechst 33342 for 5 minutes. The sections were then mounted on microscope slides and examined under ultraviolet light using an epifluorescence microscope. Ten visual fields were analyzed per retina; the experiment was replicated three times.

\section{Western blot}

Protein extracts were generated from retinas. Retinas from sham operation group ( $\mathrm{Ctrl}$ ), $\mathrm{AOH}$ group, $\mathrm{AOH}+\mathrm{PBS}$ control group, and $\mathrm{AOH}+\mathrm{AAA}$ group were homogenized in lysis buffer and sonicated to dissolve the tissue completely. In all, $40 \mu \mathrm{g}$ of total proteins from each sample were loaded per lane for sodium dodecyl sulfate polyacrylamide gel electrophoresis. The proteins were transferred onto the nitrocellulose membrane (Schleicher \& Schuell, Dassel, Germany) 
and the blocked nitrocellulose membrane was incubated with a primary rabbit polyclonal antibody against TNF- $\alpha(1: 1,000$; Abcam) for 3 hours (room temperature). After washing in Tris Buffered Saline, with Tween-20 (TBST), the membranes were incubated with horseradish peroxidase-conjugated goat anti-rabbit or mouse IgG prior to detection of the labeled proteins by using ECL-plus kit (PerkinElmer Inc., Waltham, MA, USA) followed by exposure of blots to X-Omat (Kodak, Rochester, NY, USA) imaging film. The images were quantified using ImageJ software (National Institutes of Health, Maryland, USA). The Gel Doc-2000 imaging system (Bio-Rad Laboratories Inc., Hercules, CA, USA) was used to perform the quantitative analysis of Western blot results. The ratio of TNF- $\alpha$ was determined by normalizing with the levels of endogenous $\beta$-actin. The $\beta$-actin band density was expressed as $100 \%$, and the other group was expressed as percentage of that from the control group.

\section{Statistical analysis}

The normality of the data was tested using the Shapiro-Wilk method. Analysis of variance was used to determine the difference between TNF- $\alpha$ and fluorescence intensity among three or more independent (unrelated) groups, followed by multiple comparisons using the Student-Newman-Keuls test. The Kruskal-Wallis test was used to determine the difference between TUNEL-positive cells and Thy-1-positive RGCs among three or more independent (unrelated) groups, followed by multiple comparisons using the Nemenyi test. Statistical analysis was performed using SAS 9.2 (SAS
Institute Inc., Cary, NC, USA). Values were reported as mean $\pm \mathrm{SD}$. A value of $P<0.05$ was considered as statistically significant.

\section{Results \\ AAA attenuates $\mathrm{AOH}$-induced cell apoptosis in RGCs}

To determine the effects of AAA on RGCs in the AOH model, $\mathrm{AOH}$ was induced in SD rats and rats were intravitreally treated with PBS, AAA, AOH + PBS, and AOH + AAA. Significantly reduced retinal thickness in the inner plexiform layer (IPL) and inner nuclear layer was observed after $\mathrm{AOH}$ induction versus control (Figure S1). TUNEL assay was used to examine the cell apoptosis in the retinal sections. The mean values of TUNEL-positive cells per visual field were $0.1 \pm 0.3$ in Control (Figure 1A), 0.2 \pm 0.4 in AAA(Figure 1B), 4.6 \pm 0.8 in $\mathrm{AOH}+\mathrm{PBS}$, and $1.7 \pm 0.7$ in $\mathrm{AOH}+\mathrm{AAA}$. AOH induced the apoptotic death of cells in the ganglion cell layer(GCL; Figure $1 \mathrm{C}$ ). In the $\mathrm{AOH}$ model, intravitreal treatment with AAA attenuated the TUNEL-positive RGCs more significantly than the intravitreal treatment with PBS $(P<0.05$; Figure 1D), indicating that AAA treatment rescued RGCs from the cell apoptosis induced in the AOH model.

\section{AAA preserves the $\mathrm{AOH}$-reduced number of RGCs}

In addition, retinal sections were immunostained with antibody against Thy-1, a marker for RGCs. ${ }^{25}$ The mean values of Thy-1-positive RGCs per visual field in Ctrl, AAA,
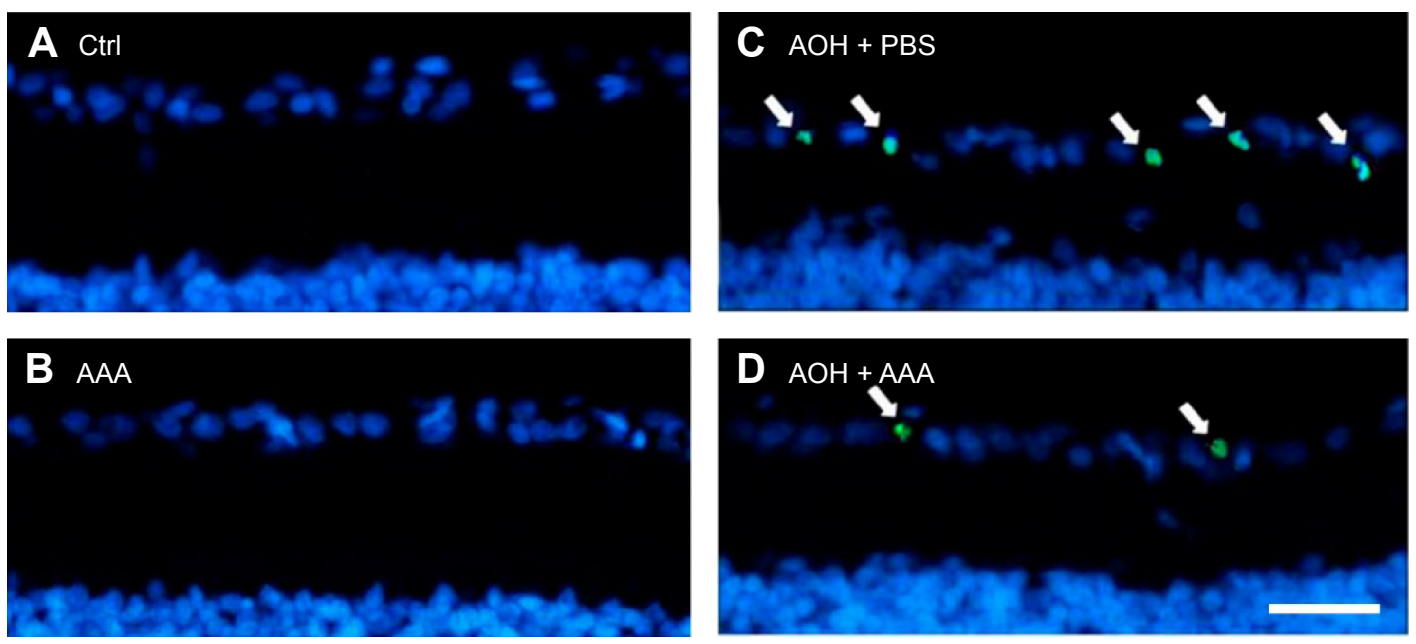

Figure I AAA attenuates $\mathrm{AOH}$-induced retinal apoptosis.

Notes: TUNEL (A-D) stainings were performed after 5 days of $\mathrm{AOH}$. Cell nuclei were counterstained with Hoechst. The results showed that AOH caused significant increase in TUNEL-positive cells ( $\mathrm{C}$ and $\mathrm{I}$ ), but AAA could abolish this effect (scale bar $=50 \mu \mathrm{m} ; \mathrm{n}=4$ per group). The green dot indicated by the arrow is an apoptotic cell and the blue areas indicate cell nuclei.

Abbreviations: AAA, $\alpha$-aminoadipic acid; $\mathrm{AOH}$, acute ocular hypertension; TUNEL, terminal deoxynucleotidyl transferase-mediated uridine $5^{\prime}$-triphosphate-biotin nick end labeling; Ctrl, control. 

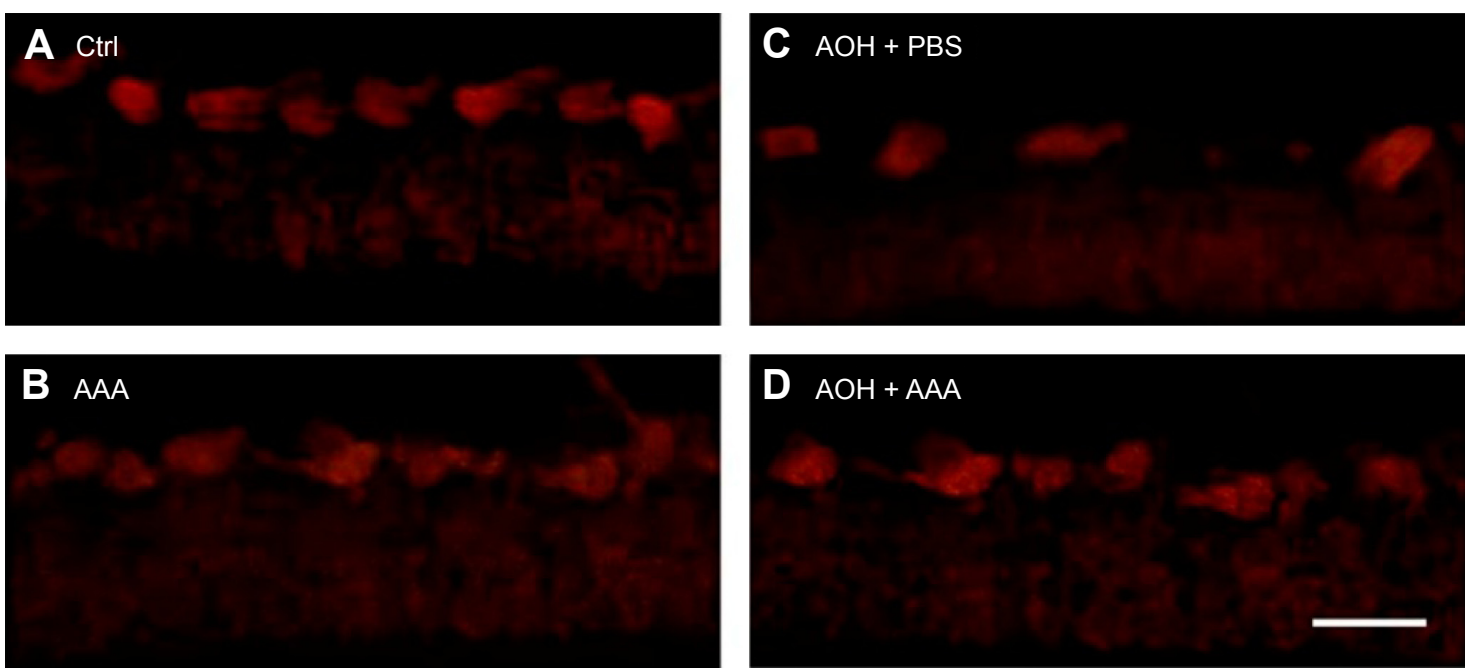

Figure 2 AAA rescued $A O H$-reduced number of RGCs.

Notes: Retinas were collected after 5 days of $A O H$, and Thy-I staining was performed. Cell nuclei were counterstained with Hoechst. The number of Thy-I-positive RGCs was quantified. In the rat model of $A O H$, the number of RGCs was significantly decreased and AAA restored the number of RGCs (scale bar $=50 \mu m$; $n=4$ per group). Abbreviations: AAA, $\alpha$-aminoadipic acid; AOH, acute ocular hypertension; RGCs, retinal ganglion cells; Ctrl, control; PBS, phosphate-buffered saline.

$\mathrm{AOH}+\mathrm{PBS}$, and $\mathrm{AOH}+\mathrm{AAA}$ groups were $8.2 \pm 0.9,8.1 \pm 0.8$ $3.9 \pm 0.7$, and $6 \pm 0.6$, respectively. AAA treatment did not change the number of RGCs in normal conditions (Figure 2A and B). When compared with the Ctrl group (Figure 2A), the number of Thy-1-positive RGCs was decreased in the AOH + PBS group (Figure 2C), and AAA treatment significantly rescued Thy-1-positive RGCs in $\mathrm{AOH}(P<0.05$; Figure 2D), corresponding to AAA-attenuated apoptosis of RGCs in AOH $(\mathrm{AOH}+\mathrm{PBS}$ versus $\mathrm{AOH}+\mathrm{AAA}, P<0.05$; Figure 1$)$.

\section{AAA attenuates $\mathrm{AOH}$-induced Müller cell gliosis}

In the rat model of AOH, AAA rescued RGCs. To determine if AAA protects against $\mathrm{AOH}$-induced RGC death through modulating Müller cell gliosis, immonohistochemistry for GFAP (a marker for activated glial cells) and GS (Müller cell-specific marker) was conducted. ${ }^{26}$ Müller cells cover the whole retina from the nerve fiber layer to the photoreceptor layer, ${ }^{27}$ and astrocytes mainly locate along GCL. GFAP was mainly expressed along nerve fiber layer and GCL and was less or absent at other layers in the control group (Figure S2). In the rat model of AOH, colocalization of GFAP and GS was observed across IPL (Figure S2), indicating Müller cell activation. After 1 day, 3 days, and 5 days of $\mathrm{AOH}$ induction in rat, GFAP expression was also observed across IPL (Figure S3), indicating Müller cell gliosis. AAA treatment versus PBS control markedly reduced GFAP immunoreactivity across IPL (Figure 3), indicating attenuation in Müller cell gliosis.

\section{AAA decreases $\mathrm{AOH}$-induced TNF- $\alpha$ production}

As mentioned earlier, AAA rescued RGCs likely through the inhibition of Müller cells gliosis in the rat model with $\mathrm{AOH}$. Activation of Müller cells may induce TNF- $\alpha$ production, leading to neurodegeneration in glaucoma. ${ }^{10}$ To determine if AAA modulates TNF- $\alpha$ production, Western blot was
A

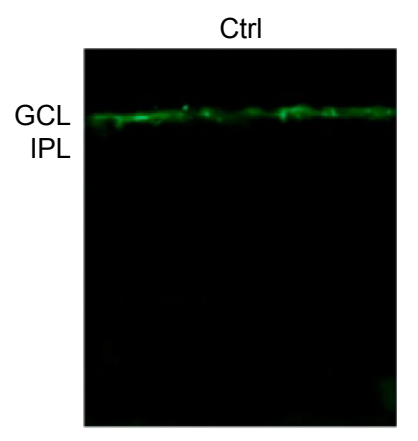

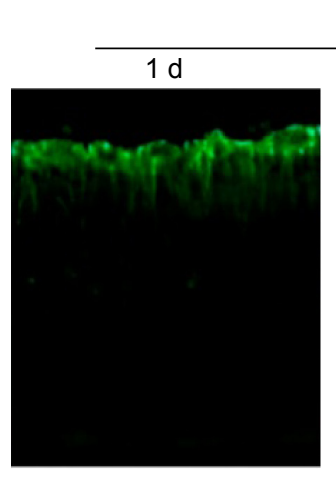

After $\mathbf{A O H}$

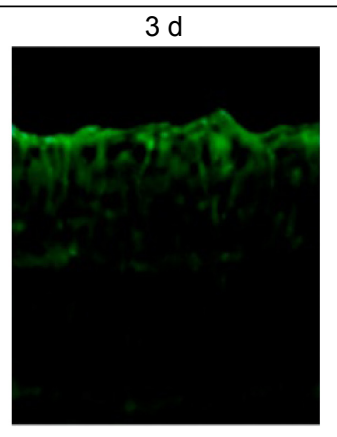

$5 d$

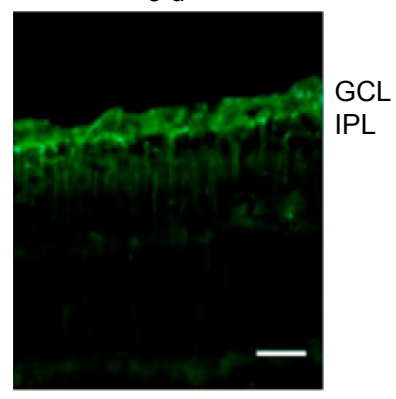

Figure 3 (Continued) 


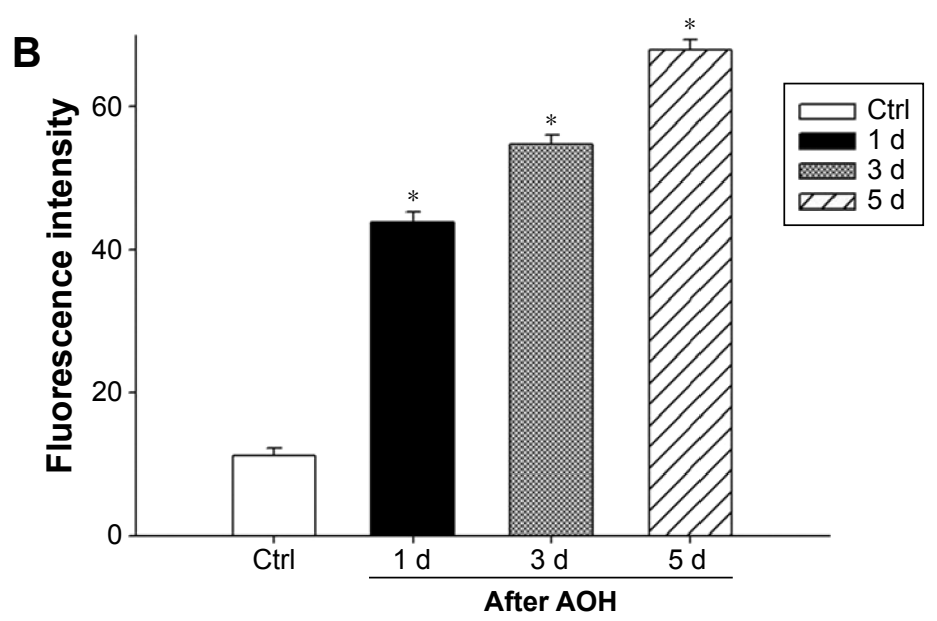

Figure 3 AAA attenuated induced Müller gliosis in the rat model of $\mathrm{AOH}$.

Notes: (A) AAA attenuated increased GFAP immunoreactivity in the Müller cells of retina of the rat model of AOH after I d, $3 \mathrm{~d}$, and $5 \mathrm{~d}$ of intravitreal AAA treatment

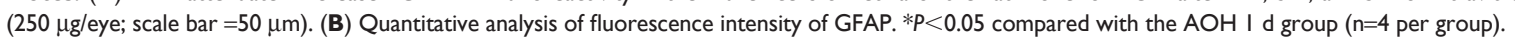

Abbreviations: AAA, $\alpha$-aminoadipic acid; AOH, acute ocular hypertension; GFAP, glial fibrillary acidic protein; d, day; Ctrl, control; GCL, ganglion cell layer; IPL, inner plexiform layer.

performed for the investigation of retinal protein levels of TNF- $\alpha$. Significantly upregulated protein levels of retinal TNF- $\alpha$ were observed in $\mathrm{AOH}$ versus sham operation $(\mathrm{Ctrl})$ group $(P<0.05$; Figure 4). Importantly, AAA treatment versus PBS control significantly attenuated TNF- $\alpha$ levels in the retinas of the rat model of $\mathrm{AOH}(P<0.05$; Figure 4$)$.

\section{Discussion}

Glaucoma is a leading cause of irreversible vision loss characterized by progressive death of RGCs, and elevated IOP is a major risk factor. ${ }^{28} \mathrm{~A}$ rodent model of $\mathrm{AOH}$ is well established for acute angle closure glaucoma, and it has been widely used to investigate the pathogenesis of death of RGCs. ${ }^{29}$ In the

A
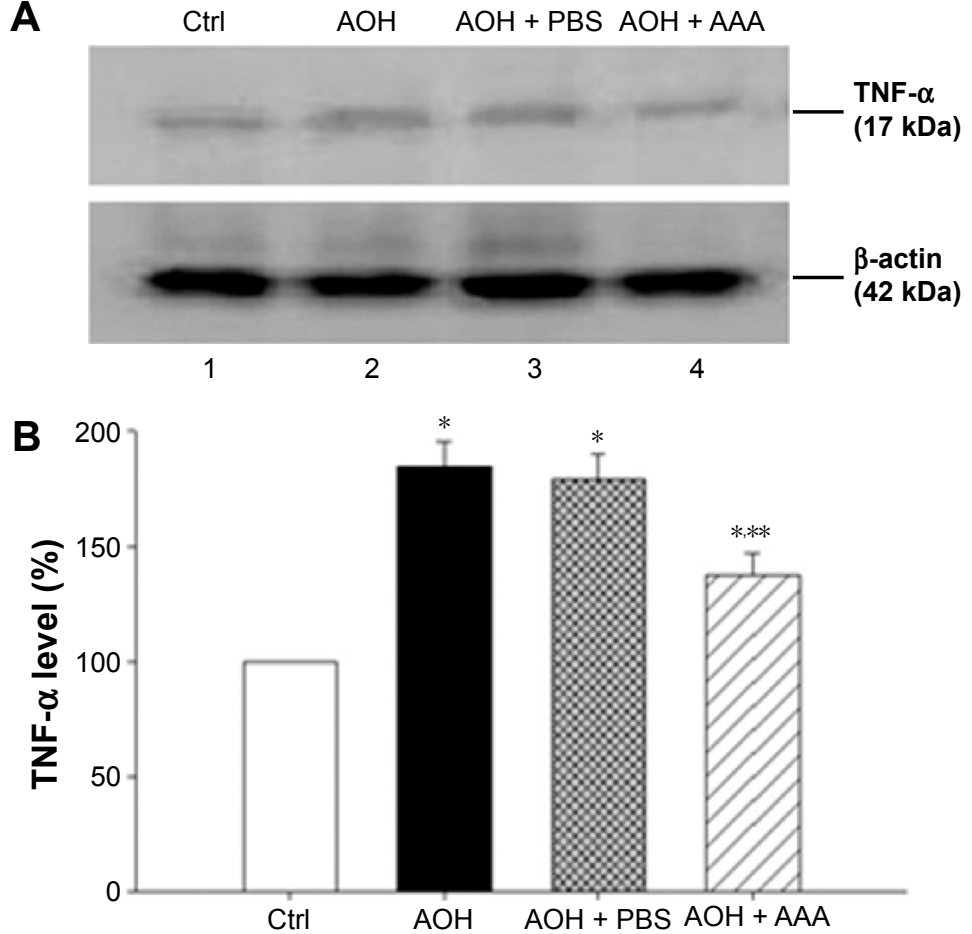

Figure 4 AAA treatment reduced the induction of TNF- $\alpha$ in $A O H$ retinas.

Notes: (A) Western blot results showed the effect of AAA on TNF- $\alpha$ protein expression in retinas after $5 \mathrm{~d}$ of AOH. (B) Quantitative analysis of protein expression levels of TNF- $\alpha$ ( $n=4$ per group). ${ }^{*} P<0.05$ compared with the Ctrl group (no $A O H$ induction). ${ }^{*} * P<0.05$ compared with the $A O H+P B S$ group.

Abbreviations: AAA, $\alpha$-aminoadipic acid; TNF- $\alpha$, tumor necrosis factor- $\alpha$; $d$, days; AOH, acute ocular hypertension; Ctrl, control; PBS, phosphate-buffered saline. 


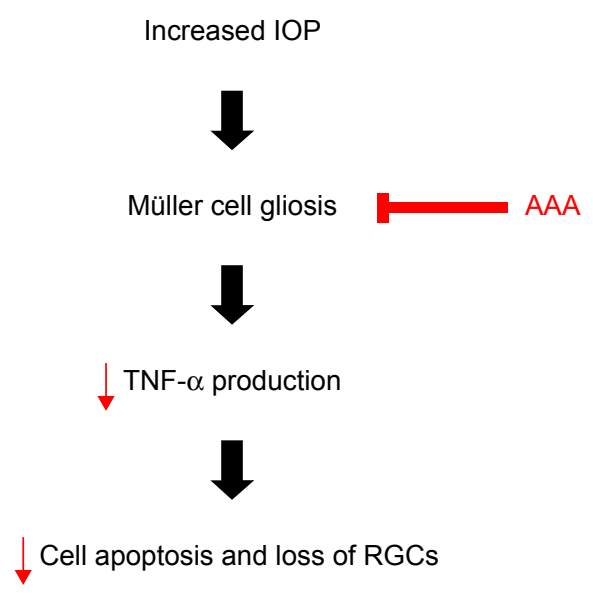

Figure 5 A schematic flow chart of AAA protection in the rat model of $\mathrm{AOH}$. Notes: In the rat model of $\mathrm{AOH}$, acute elevation of IOP induced Müller cell gliosis and TNF- $\alpha$ production, leading to RGC apoptosis. AAA treatment reduced Müller cell gliosis and TNF- $\alpha$ production to protect RGCs.

Abbreviations: AAA, $\alpha$-aminoadipic acid; $\mathrm{AOH}$, acute ocular hypertension; IOP, intraocular pressure; RGC, retinal ganglion cells, TNF- $\alpha$, tumor necrosis factor- $\alpha$.

current rat model of $\mathrm{AOH}$, we found induced cell apoptosis and decreased number of RGCs with IOP induction versus sham operation control. Importantly, treatment of AAA versus PBS control significantly attenuated RGC apoptosis and rescued the reduced number of RGCs, demonstrating protective effects on $\mathrm{AOH}$ retinas (Figure 5).

Activated Müller cells contribute to the progression of glaucoma. ${ }^{30}$ Müller cells perform a multitude of important regulatory and supportive roles, including secretion of trophic factors, removal of metabolic waste, and neurotransmitter recycling. ${ }^{31,32} \mathrm{We}$ found that Müller cells were activated with induced GFAP immunoreactivity in the rat model of $\mathrm{AOH}$ versus control retinas. GFAP, an intermediate filament protein, is considered as a marker of reactive Müller cell gliosis, ${ }^{27}$ which is not or less expressed in Müller cells in normal retinas and expressed highly at ischemic, ${ }^{33}$ light-induced retinal degeneration, ${ }^{34}$ and retinal detachment. ${ }^{35}$ Activation of Müller cells so far was demonstrated to have both protective and detrimental effects. ${ }^{36-38}$ Especially early after injury, Müller cell gliosis is believed to be neuroprotective and promotes the repair of neurons in response to injury, ${ }^{36}$ with GFAP upregulation to provide additional structural integrity to the retina at the site of injury. ${ }^{39}$ However, activated Müller cells also express TNF- $\alpha,{ }^{40}$ monocyte chemoattractant protein, ${ }^{41}$ and nitric oxide ${ }^{42}$ to induce death of RGCs. TNF- $\alpha$ induction contributes to inflammation, apoptosis, and necrosis, leading to cell death. We observed that AAA treatment attenuated GFAP immunoreactivity in Müller cell processes across IPL and reduced retinal TNF- $\alpha$ levels in the rat model of $\mathrm{AOH}$. These findings suggested that AAA promoted survival of RGCs in the rat model of $\mathrm{AOH}$, likely through the inhibition of $\mathrm{AOH}$-induced Müller cell gliosis and in turn downregulation of TNF- $\alpha$ protein production.

\section{Conclusion}

We found that AAA could effectively protect retina against loss of RGCs and apoptosis in $\mathrm{AOH}$ retinas through attenuating Müller cell gliosis and downregulating TNF- $\alpha$ production. These observations suggested that AAA might be a potential therapeutic target in the treatment of neurodegeneration in glaucoma.

\section{Acknowledgments}

This study was funded by Beijing Municipal Administration of Hospitals Clinical Medicine Development of Special Funding Support (No XM201305) and Clinical-Basic Cooperation Program from Capital Medical University (Nos 14JL32 and 2015YKSJ02).

\section{Disclosure}

The authors report no conflicts of interest in this work.

\section{References}

1. Gugleta K, Turksever C, Polunina A, Orgul S. Effect of ageing on the retinal vascular responsiveness to flicker light in glaucoma patients and in ocular hypertension. Br J Ophthalmol. 2013;97(7):848-851.

2. Husain S, Abdul Y, Crosson CE. Preservation of retina ganglion cell function by morphine in a chronic ocular-hypertensive rat model. Invest Ophthalmol Vis Sci. 2012;53(7):4289-4298.

3. Ren R, Jonas JB, Tian G, et al. Cerebrospinal fluid pressure in glaucoma: a prospective study. Ophthalmology. 2010;117(2):259-266.

4. Chung SH, Shen W, Jayawardana K, et al. Differential gene expression profiling after conditional Muller-cell ablation in a novel transgenic model. Invest Ophthalmol Vis Sci. 2013;54(3):2142-2152.

5. Coorey NJ, Shen W, Chung SH, Zhu L, Gillies MC. The role of glia in retinal vascular disease. Clin Exp Optom. 2012;95(3):266-281.

6. Tezel G; Fourth ARVO/Pfizer Ophthalmics Research Institute Conference Working Group. The role of glia, mitochondria, and the immune system in glaucoma. Invest Ophthalmol Vis Sci. 2009;50(3): 1001-1012.

7. Johnson EC, Morrison JC. Friend or foe? Resolving the impact of glial responses in glaucoma. J Glaucoma. 2009;18(5):341-353.

8. Bringmann A, Wiedemann P. Muller glial cells in retinal disease. Ophthalmologica. 2012;227(1):1-19.

9. Wang X, Ng YK, Tay SS. Factors contributing to neuronal degeneration in retinas of experimental glaucomatous rats. J Neurosci Res. 2005;82(5):674-689.

10. Tezel G. TNF-alpha signaling in glaucomatous neurodegeneration. Prog Brain Res. 2008;173:409-421.

11. Gupta S. Molecular steps of tumor necrosis factor receptor-mediated apoptosis. Curr Mol Med. 2001;1(3):317-324.

12. Gesslein B, Hakansson G, Gustafsson L, Ekstrom P, Malmsjo M. Tumor necrosis factor and its receptors in the neuroretina and retinal vasculature after ischemia-reperfusion injury in the pig retina. Mol Vis. 2010;16:2317-2327.

13. Tezel G, Li LY, Patil RV, Wax MB. TNF-alpha and TNF-alpha receptor-1 in the retina of normal and glaucomatous eyes. Invest Ophthalmol Vis Sci. 2001;42(8):1787-1794. 
14. Gustavsson C, Agardh CD, Hagert P, Agardh E. Inflammatory markers in nondiabetic and diabetic rat retinas exposed to ischemia followed by reperfusion. Retina. 2008;28(4):645-652.

15. Nakazawa T, Nakazawa C, Matsubara A, et al. Tumor necrosis factor-alpha mediates oligodendrocyte death and delayed retinal ganglion cell loss in a mouse model of glaucoma. J Neurosci. 2006; 26(49):12633-12641.

16. Cheong CU, Chang CP, Chao CM, Cheng BC, Yang CZ, Chio CC. Etanercept attenuates traumatic brain injury in rats by reducing brain TNF-alpha contents and by stimulating newly formed neurogenesis. Mediators Inflamm. 2013;2013:620837.

17. Maddahi A, Kruse LS, Chen QW, Edvinsson L. The role of tumor necrosis factor-alpha and TNF-alpha receptors in cerebral arteries following cerebral ischemia in rat. J Neuroinflammation. 2011;8:107.

18. Pannicke T, Stabel J, Heinemann U, Reichelt W. alpha-Aminoadipic acid blocks the $\mathrm{Na}(+)$-dependent glutamate transport into acutely isolated Muller glial cells from guinea pig retina. Pflugers Arch. 1994; 429(1):140-142.

19. Ishikawa Y, Mine S. Aminoadipic acid toxic effects on retinal glial cells. Jpn J Ophthalmol. 1983;27(1):107-118.

20. Khurgel M, Koo AC, Ivy GO. Selective ablation of astrocytes by intracerebral injections of alpha-aminoadipate. Glia. 1996;16(4): 351-358.

21. Linser PJ, Moscona AA. Induction of glutamine synthetase in embryonic neural retina: its suppression by the gliatoxic agent alpha-aminoadipic acid. Brain Res. 1981;227(1):103-119.

22. Huck S, Grass F, Hatten ME. Gliotoxic effects of alpha-aminoadipic acid on monolayer cultures of dissociated postnatal mouse cerebellum. Neuroscience. 1984;12(3):783-791.

23. Rich KA, Figueroa SL, Zhan Y, Blanks JC. Effects of Muller cell disruption on mouse photoreceptor cell development. Exp Eye Res. 1995; 61(2):235-248.

24. Ganesh BS, Chintala SK. Inhibition of reactive gliosis attenuates excitotoxicity-mediated death of retinal ganglion cells. PLoS One. 2011;6(3):e18305.

25. Barnstable CJ, Drager UC. Thy-1 antigen: a ganglion cell specific marker in rodent retina. Neuroscience. 1984;11(4):847-855.

26. Chen H, Weber AJ. Expression of glial fibrillary acidic protein and glutamine synthetase by Muller cells after optic nerve damage and intravitreal application of brain-derived neurotrophic factor. Glia. 2002; 38(2):115-125.

27. Bringmann A, Iandiev I, Pannicke T, et al. Cellular signaling and factors involved in Muller cell gliosis: neuroprotective and detrimental effects. Prog Retin Eye Res. 2009;28(6):423-451.
28. Gupta N, Yucel YH. Glaucoma as a neurodegenerative disease. Curr Opin Ophthalmol. 2007;18(2):110-114.

29. Mi XS, Feng Q, Lo AC, et al. Protection of retinal ganglion cells and retinal vasculature by Lycium barbarum polysaccharides in a mouse model of acute ocular hypertension. PLoS One. 2012;7(10):e45469.

30. Chong RS, Martin KR. Glial cell interactions and glaucoma. Curr Opin Ophthalmol. 2015;26(2):73-77.

31. Bringmann A, Pannicke T, Grosche J, et al. Muller cells in the healthy and diseased retina. Prog Retin Eye Res. 2006;25(4):397-424.

32. Reichenbach A, Bringmann A. New functions of Muller cells. Glia. 2013; 61(5):651-678.

33. Wurm A, Iandiev I, Uhlmann S, et al. Effects of ischemia-reperfusion on physiological properties of Muller glial cells in the porcine retina. Invest Ophthalmol Vis Sci. 2011;52(6):3360-3367.

34. Torbidoni V, Iribarne M, Suburo AM. Endothelin receptors in lightinduced retinal degeneration. Exp Biol Med (Maywood). 2006;231(6): 1095-1100.

35. Ghosh F, Johansson K. Neuronal and glial alterations in complex long-term rhegmatogenous retinal detachment. Curr Eye Res. 2012; 37(8):704-711.

36. Kim JH, Kim JH, Park JA, et al. Blood-neural barrier: intercellular communication at glio-vascular interface. J Biochem Mol Biol. 2006; 39(4):339-345.

37. Bringmann A, Pannicke T, Biedermann B, et al. Role of retinal glial cells in neurotransmitter uptake and metabolism. Neurochem Int. 2009; 54(3-4):143-160.

38. Martin KR, Levkovitch-Verbin H, Valenta D, Baumrind L, Pease ME, Quigley HA. Retinal glutamate transporter changes in experimental glaucoma and after optic nerve transection in the rat. Invest Ophthalmol Vis Sci. 2002;43(7):2236-2243.

39. Dyer MA, Cepko CL. Control of Muller glial cell proliferation and activation following retinal injury. Nat Neurosci. 2000;3(9):873-880.

40. Lei X, Zhang J, Shen J, et al. EPO attenuates inflammatory cytokines by Muller cells in diabetic retinopathy. Front Biosci (Elite Ed). 2011;3: 201-211.

41. Nakazawa T, Hisatomi T, Nakazawa C, et al. Monocyte chemoattractant protein 1 mediates retinal detachment-induced photoreceptor apoptosis. Proc Natl Acad Sci U S A. 2007;104(7):2425-2430.

42. Zeng K, Xu H, Chen K, et al. Effects of taurine on glutamate uptake and degradation in Muller cells under diabetic conditions via antioxidant mechanism. Mol Cell Neurosci. 2010;45(2):192-199. 


\section{Supplementary materials}

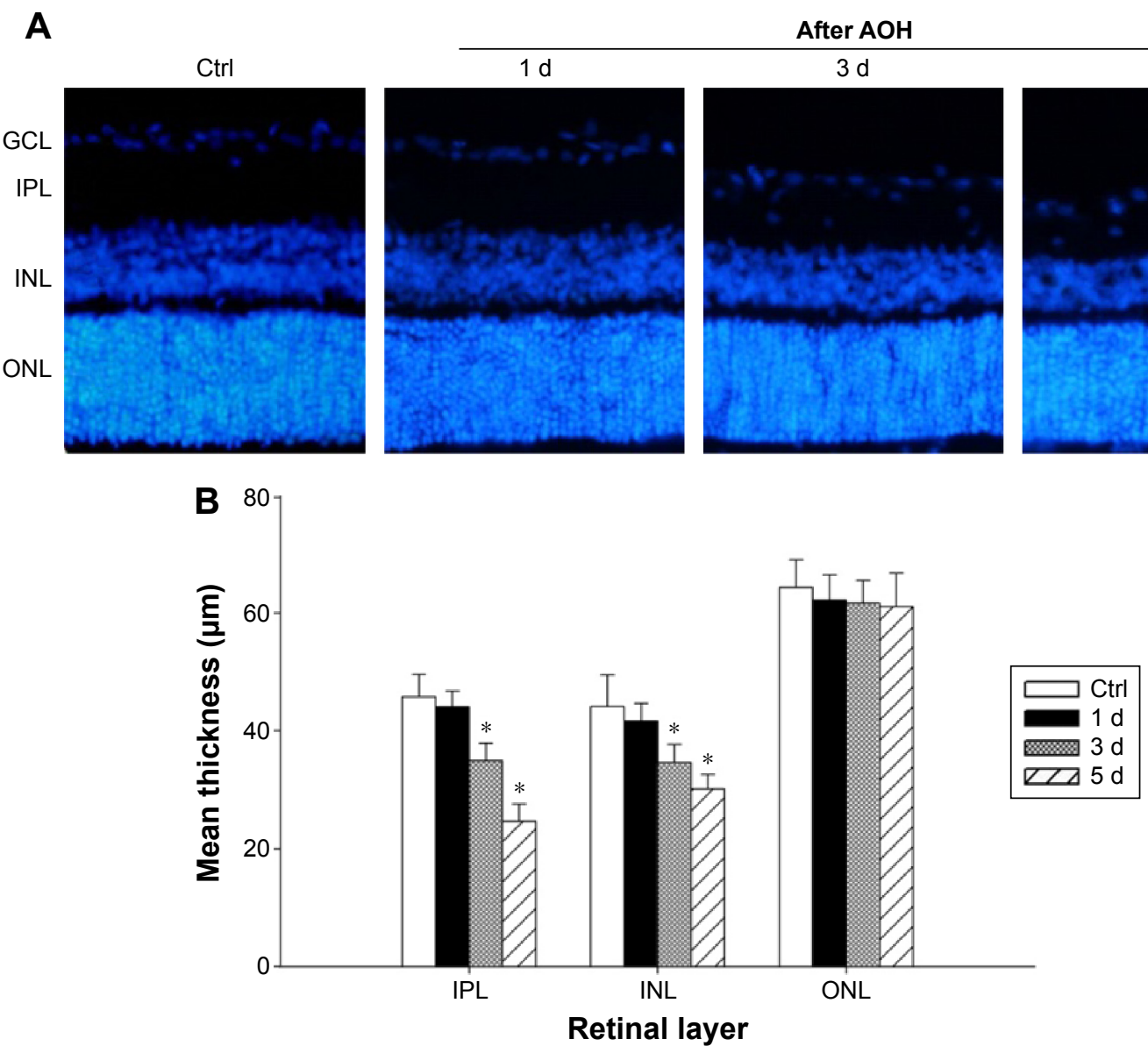

Figure SI (A) Reprehensive results of Hoechst staining in rat retina after I d, $3 \mathrm{~d}$, and $5 \mathrm{~d}$ of $\mathrm{AOH}$; (B) Quantitative analysis of the retinal thickness.

Notes: The results showed that the thickness of IPL and INL layers decreased significantly following the reperfusion time after $A O H$ treatment $($ scale bar $=50 \mu m)$. $* P<0.05$ compared with the control group ( $\mathrm{n}=4$ per group).

Abbreviations: AOH, acute ocular hypertension; IPL, inner plexiform layer; INL, inner nuclear layer; Ctrl, control; d, day; GCL, ganglion cell layer; ONL, outer nuclear layer.

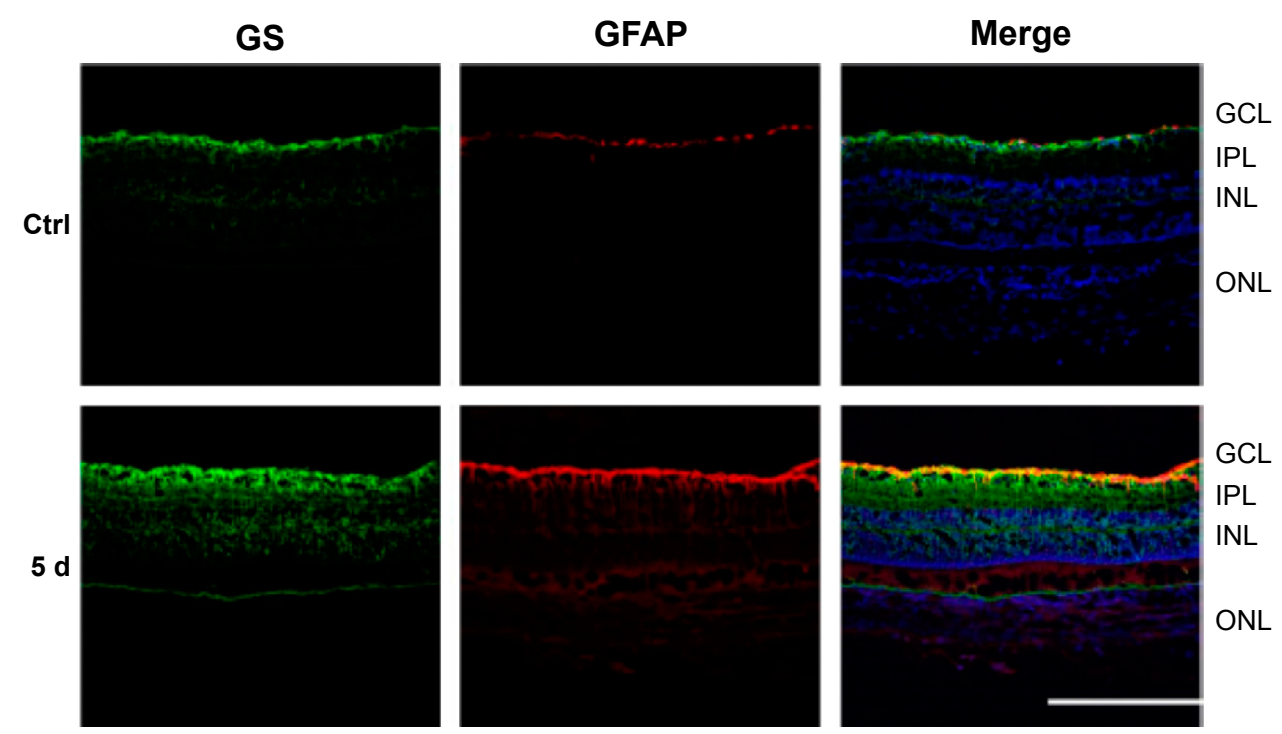

Figure S2 Immunohistochemistry costained with antibodies against GS (green) and GFAP (red).

Note: Strong expression of GFAP was shown in the GS-positive Müller cell after $5 \mathrm{~d}$ of AOH compared with the control group (scale bar $=50 \mu \mathrm{m}$ ).

Abbreviations: GS, glutamine synthetase; GFAP, glial fibrillary acidic protein; AOH, acute ocular hypertension; Ctrl, control; GCL, ganglion cell layer; IPL, inner plexiform layer; INL, inner nuclear layer; ONL, outer nuclear layer; d, days. 


\section{A \\ A}

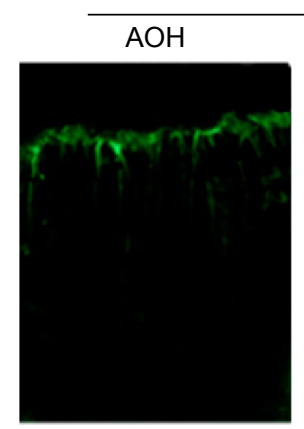

$1 \mathrm{~d}$
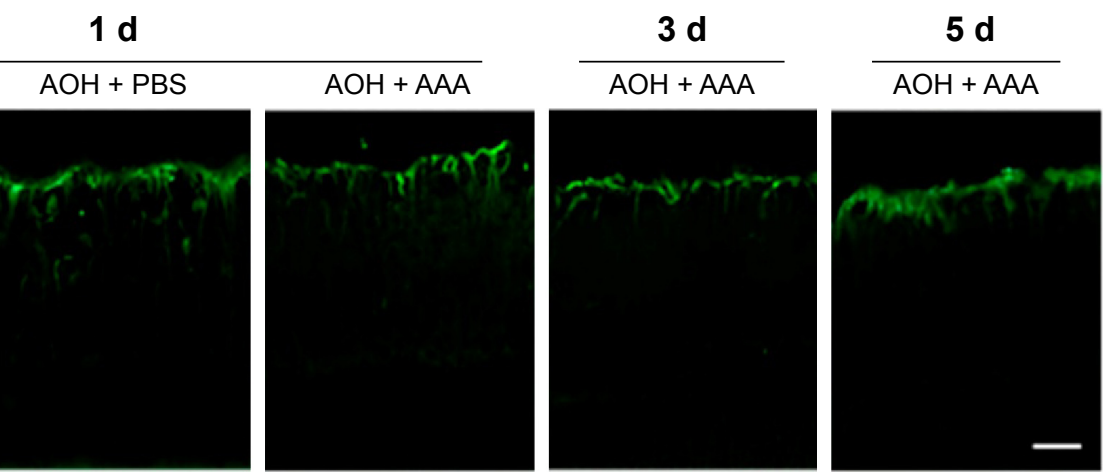

B

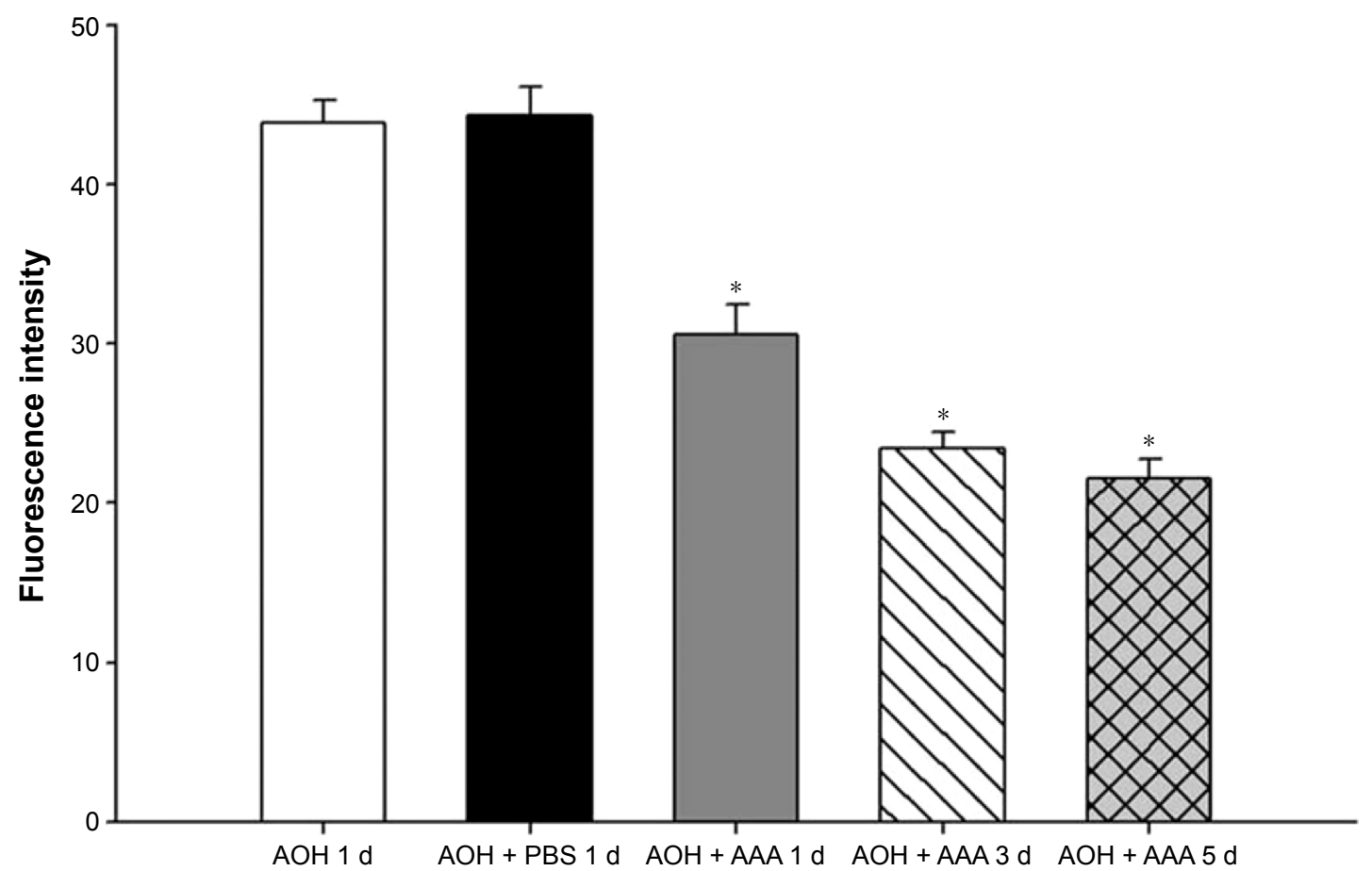

Figure S3 Increased GFAP immunoreactivity after $\mathrm{AOH}$ induction in rat retinas.

Notes: (A) Immunofluorescent staining showed that AOH increased GFAP immunoreactivity in the Müller cell processes across IPL. Scale bar $=50 \mu \mathrm{m}$. $(\mathbf{B})$. Quantitative analysis of fluorescence intensity of GFAP ( $n=4$ per group). $* P<0.05$ compared with the control group.

Abbreviations: GFAP, glial fibrillary acidic protein; AOH, acute ocular hypertension; IPL, inner plexiform layer; d, day; PBS, phosphate-buffered saline; AAA, $\alpha$-aminoadipic acid.

\section{Publish your work in this journal}

Drug Design, Development and Therapy is an international, peerreviewed open-access journal that spans the spectrum of drug design and development through to clinical applications. Clinical outcomes, patient safety, and programs for the development and effective, safe, and sustained use of medicines are the features of the journal, which has also been accepted for indexing on PubMed Central. The manuscript management system is completely online and includes a very quick and fair peer-review system, which is all easy to use. Visit http://www.dovepress.com/testimonials.php to read real quotes from published authors. 